\title{
Parathyroidectomy for Refractory Secondary Hyperparathyroidism with Severe Bone Disease
}

\author{
Teodora Amor N. Evora and Roberto C. Mirasol
}

Section of Endocrinology and Metabolism, St. Luke's Medical Center, Quezon City, Philippines

\begin{abstract}
Advances in the medical management of secondary hyperparathyroidism due to renal failure have decreased the use of parathyroidectomy as a treatment option. However, some patients with end-stage renal disease still progress to refractory hyperparathyroidism and debilitating bone disease, for which parathyroidectomy may be warranted. We describe a case of a 35-year-old female on chronic hemodialysis who presented with bone pains, deformities, decrease in height and recurrent pathologic fractures. She had markedly elevated parathyroid hormone (PTH), vitamin D deficiency, persistent hyperphosphatemia and hypocalcemia despite therapeutic measures. Subtotal parathyroidectomy was done with eventual improvement of biochemical abnormalities, resolution of bone pains and healing of fractures.
\end{abstract}

Keywords: secondary hyperparathyroidism, hyperparathyroid bone disease, subtotal parathyroidectomy

\section{Introduction}

Chronic kidney disease (CKD) is a growing public health concern worldwide. In the Philippines, the prevalence of CKD with glomerular filtration rate (GFR) of 60 to 89 $\mathrm{mL} / \mathrm{min}$ is $24.6 \%$, while that of GFR less than $60 \mathrm{~mL} / \mathrm{min}$ is $2.6 \%$. This indicates that in 2005, over one million Filipinos had end stage CKD. ${ }^{1}$ While efforts to delay the progression of kidney disease are fundamental, it is also imperative to initiate early and aggressive management of its complications to reduce morbidity and mortality.

Secondary hyperparathyroidism (SHPT) is one complication of CKD that develops early in the course of the disease. When left untreated, SHPT leads to bone disease that may cause pain, fractures and disability. Unfortunately, SHPT is often unrecognized and inadequately treated in patients with early stages of kidney disease, when medical therapy would have greater benefits. ${ }^{2}$ Some patients with late stage CKD requiring dialysis would already have developed refractory SHPT with renal osteodystrophy. These patients may benefit from surgical treatment to prevent or alleviate its consequences.

\section{Case report}

A 35-year-old female complained of chronic low back and right hip pain with deformities of the chest, back, and distal forearm. She also noted a gradual decrease in height by about 10 centimeters. She was diagnosed with CKD stage 5 due to chronic pyelonephritis secondary to

ISSN 0857-1074

Printed in the Philippines

Copyright $($ C 2012 by the JAFES

Received September 15, 2011. Accepted April 11, 2012. nephrolithiasis. She underwent right nephrectomy 8 years prior to consultation. Subsequently, she was maintained on hemodialysis 2 times a week, calcium-based phosphate binders, calcium and cholecalciferol supplementation, antihypertensive medications, erythropoietin injections and iron with folic acid. Physical examination revealed kyphoscoliosis of the thoracic spine and deformity of the right wrist without any known history of trauma.

Diagnostic tests revealed a markedly elevated intact PTH (4287 pg/mL, 65 times the upper limit of normal) and vitamin D deficiency (25-hydroxycholecalciferol 17.32 $\mathrm{ng} / \mathrm{mL})$. Serum phosphorus was elevated (5.9 mg/dL) and ionized calcium was low (0.95 mmol/L) (Table 1). On ultrasonography, parathyroid nodules were found bilaterally. In contrast, parathyroid scintigraphy did not show any sestamibi-avid lesions in the anterior neck.

Skeletal x-rays showed compression deformities of the thoracic and lumbar vertebrae, thoraco-lumbar levoscoliosis with decrease in vertebral height and decrease in bone mineralization signifying osteopenia (Figures 1A and B). Pelvic x-rays revealed asymmetry of the pubic bone with non-delineation of the symphysis pubis, and irregularity of the left inferior pubic ramus, interpreted as a pathologic fracture.

Bone densitometry was deferred following the patient's hospitalization for hip pain after alighting from a vehicle. She sustained a complete, displaced fracture of the right femoral neck. She underwent multiple hip screw fixation

Corresponding author: Teodora Amor N. Evora, MD

Mailing address: Diabetes, Thyroid and Endocrine Center

$12^{\text {th }}$ fl., Cathedral Heights Building,

St. Luke's Medical Center, E. Rodriquez Ave.,

Quezon City, Philippines

Telephone number: 7230101 (local 5210)

Fax number: 7230101 (local 5210)

Email address: amorevora@gmail.com 


\begin{tabular}{|c|c|c|c|c|c|c|}
\hline Test & Normal values & Initial & Follow-up & $\begin{array}{l}\text { Immediately } \\
\text { pre-surgery }\end{array}$ & $\begin{array}{l}\text { Immediately } \\
\text { post-surgery }\end{array}$ & $\begin{array}{c}\text { Six months } \\
\text { post-surgery }\end{array}$ \\
\hline Intact PTH & $12.00-65.00 \mathrm{pg} / \mathrm{mL}$ & 4287 & 2938 & 2725 & 98.3 & 785.5 \\
\hline Total calcium & $8.5-10.1 \mathrm{mg} / \mathrm{dL}$ & 9.2 & 9.2 & & 7.3 & 5.8 \\
\hline Ionized calcium & $1.00-1.30 \mathrm{mmol} / \mathrm{L}$ & 0.95 & 1.01 & 1.05 & 0.87 & \\
\hline Serum phosphorus & $2.5-4.9 \mathrm{mg} / \mathrm{dL}$ & 5.9 & 6.4 & 5.6 & 3.0 & 4.8 \\
\hline $25-\mathrm{OH}$ vitamin D & $\geq 30 \mathrm{ng} / \mathrm{mL}$ & 17.32 & $\ldots$ & 14.71 & $\ldots$ & 37.4 \\
\hline Serum albumin & $34-48 \mathrm{~g} / \mathrm{L}$ & 36.9 & 41.5 & $\ldots$ & 37.0 & 42.4 \\
\hline
\end{tabular}

(Figure 2). A week after the procedure, she sustained another complete fracture at the right subtrochanteric area inferior to the initial fracture site. This was managed with the application of a one and a half hip spica.

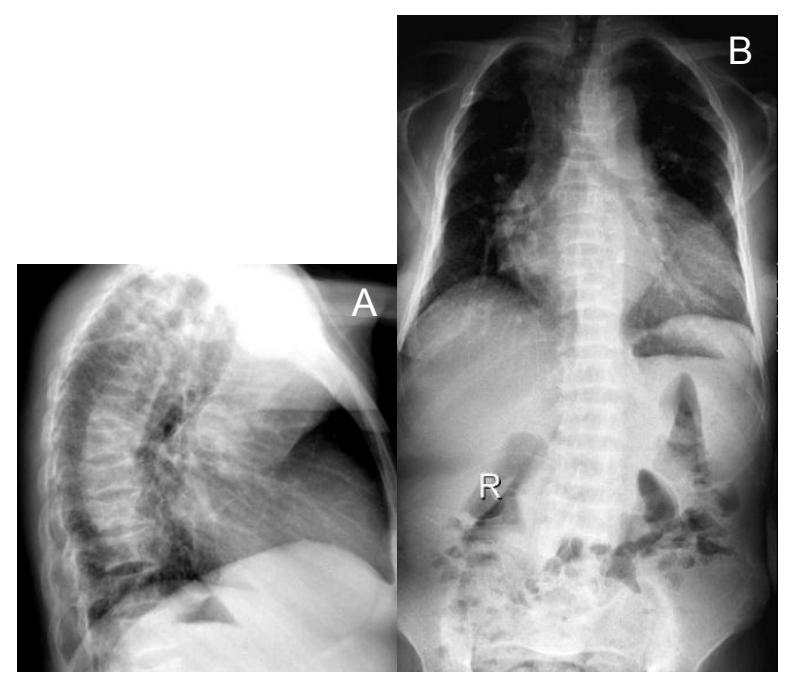

Figure 1. Radiographs of the spine. A, Thoracic spine in lateral view showing decrease in height of some of the vertebral bodies with inadequate mineralization. $B$, Thoraco-lumbar spine in anteroposterior view showing decrease in vertebral body height, leftward deviation and inadequate mineralization. $R$, Marker for right side.

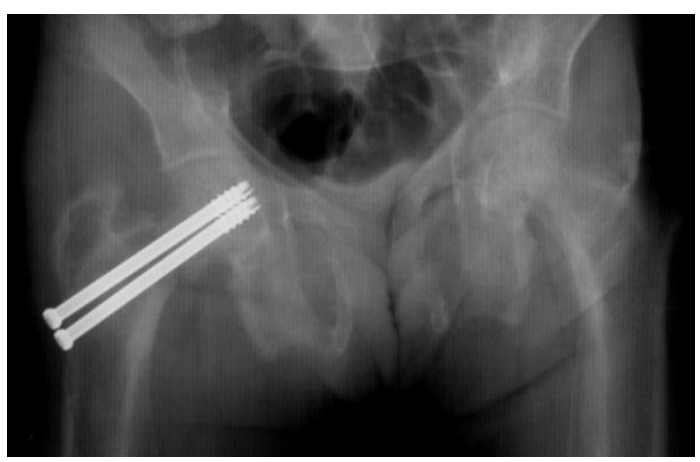

Figure 2. Radiograph of the right hip showing complete fracture of the femoral neck with multiple hip screws in place.

Calcitriol was initiated in addition to the patient's treatment regimen. Cinacalcet, a calcimimetic, was also prescribed but was not taken because it was both expensive and not available. On follow up, her intact PTH decreased and calcium level normalized, but hyperphosphatemia and vitamin D deficiency persisted (Table 1). Bone densitometry demonstrated bone mineral density measurements at the spine, femur and forearm below the expected range for the patient's age (Table 2).

\begin{tabular}{|c|c|c|c|}
\hline Scan site & Region & $\operatorname{BMD}\left(\mathrm{g} / \mathrm{cm}^{2}\right)$ & z score \\
\hline Spine AP & $\mathrm{L}_{1}-\mathrm{L}_{4}$ & 0.437 & -5.8 \\
\hline Dual femur & Total left & 0.546 & -3.4 \\
\hline Left forearm & Radius $33 \%$ & 0.576 & -3.0 \\
\hline
\end{tabular}

The patient's presentation and diagnostic test results were attributed to late manifestations of long-standing secondary hyperparathyroidism with severe symptomatic high-turnover bone disease, or osteitis fibrosa due to CKD. Surgical removal of the parathyroid glands was recommended.

She underwent with a subtotal parathyroidectomy which entailed removal of $3 \frac{1}{2}$ of her parathyroid glands. The parathyroid glands were enlarged, with a diameter more than $1 \mathrm{~cm}$ and a weight of more than $600 \mathrm{mg}$ each. Histopathology revealed parathyroid hyperplasia (Figure 3). Postoperatively, she developed hungry bone syndrome, which was managed successfully with intravenous calcium. She was later maintained on oral calcium, phosphate binder and vitamin D (both ergocalciferol and calcitriol). Six months after surgery, her intact PTH and serum phosphorus decreased to target levels. While her vitamin D level normalized, her calcium level remained low (Table 1). Her bone pains resolved and the fractures were healed. Through rehabilitation, the patient gradually regained mobility.

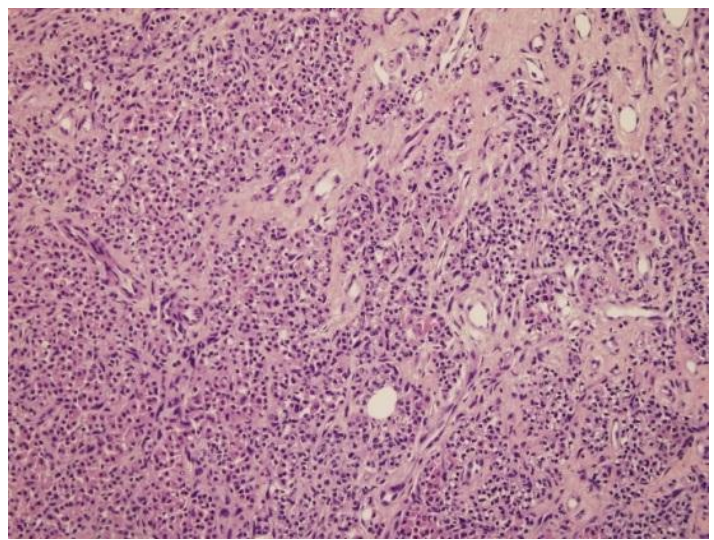

Figure 3. Photomicrograph of the parathyroid gland in hematoxylin and eosin stain at low power magnification showing diffuse hyperplasia with strands of fibrous tissue (x40). 


\section{Discussion}

SHPT begins quite early in the course of CKD, when GFR declines to less than $60 \mathrm{~mL} / \mathrm{min} / 1.73 \mathrm{~m}^{2,3}$ Therefore, screening for SHPT is recommended at stage 3 CKD (Table 3). Hyperphosphatemia in CKD plays a central role in promoting PTH release by the induction of hypocalcemia, decreased renal formation or activity of calcitriol, and by increased PTH gene expression. ${ }^{5}$

\begin{tabular}{|c|c|c|}
\hline Parameter & Target serum level & Normal range \\
\hline Intact PTH & $\begin{array}{l}\text { Within } 2-9 x \text { the upper } \\
\text { limit of normal }\end{array}$ & $12.00-65.00 \mathrm{pg} / \mathrm{mL}$ \\
\hline Phosphorous & Within normal range & $0.87-1.45 \mathrm{mmol} / \mathrm{L}$ \\
\hline Calcium & Within normal range & $8.5-10.1 \mathrm{mg} / \mathrm{dL}$ \\
\hline \multicolumn{3}{|c|}{$\begin{array}{l}\text { KDIGO, Kidney Disease Improving Global Outcomes; CKD-MBD, } \\
\text { chronic kidney disease-mineral and bone disorder } \\
\text { Source: KDIGO clinical practice guidelines for the diagnosis, evaluation, } \\
\text { prevention, and treatment of chronic kidney disease-mineral and bone } \\
\text { disorder. Kidney Int Suppl 2009;76(113):S1-S130. }\end{array}$} \\
\hline
\end{tabular}

The exact prevalence of SHPT in patients with CKD requiring dialysis (stage 5D) is not known. A US study involving 122 patients on maintenance hemodialysis for at least 12 months showed that $78 \%$ had SHPT, with a mean PTH level of $481 \mathrm{pg} / \mathrm{mL}^{6}$

Observational studies consistently report increased morbidity and mortality in patients with CKD stage 5D whose intact PTH values were greater than 9 times the upper normal limit of the assay. Once present, fully developed, severe SHPT may be resistant to pharmacologic therapy and parathyroidectomy may be required to control the mineral and hormonal disruption in SHPT. ${ }^{7}$

The initial focus in preventing and managing SHPT in the dialysis patient is the prevention and treatment of hyperphosphatemia. This may be done through dietary restriction and administration of some combination of phosphate binders (either calcium or non-calcium based), vitamin D or its analogs, and calcimimetic agents to achieve target goals (Table 3). ${ }^{7}$

Despite multiple therapeutic interventions, the patient continued to have elevated intact PTH. Refractory SHPT is due to delayed and/or inadequate therapy, persistent hyperphosphatemia, calcitriol deficiency and hypocalcemia, leading to parathyroid gland hyperplasia. In addition, somatic mutations in the proliferating parathyroid cells lead to adenomatous transformation and autonomous function. When the autonomous behavior leads to hypercalcemia, the condition is then called tertiary hyperparathyroidism. ${ }^{8}$

Intact PTH has been utilized as the best marker of bone turnover in hyperparathyroid bone disease because of the difficulty and expense of obtaining bone biopsies as the gold standard for diagnosis. Numerous studies have shown that high-turnover bone disease is often associated with intact PTH levels more than $400 \mathrm{pg} / \mathrm{mL} .{ }^{4}$ High levels of serum alkaline phosphatase generally reflect the extent of histologic changes in patients with high-turnover lesions of renal osteodystrophy and values frequently correlate with serum PTH levels. Bone-specific alkaline phosphatase activity may be useful in predicting the histologic lesions of renal osteodystrophy, but whether these values are superior to total alkaline phosphatase levels remains to be demonstrated. ${ }^{9}$

While medical therapy can effectively control SHPT in a majority of patients, it is not always successful in achieving adequate control in some. Surgery can provide marked and sustained reductions in serum PTH, calcium and phosphorus levels. ${ }^{7}$ Despite the lack of randomized, controlled trials comparing the long-term effects of medical versus surgical therapy of advanced secondary hyperparathyroidism, parathyroidectomy is generally indicated in patients with severe HPT (intact PTH more than $800 \mathrm{pg} / \mathrm{mL}$ ), associated with hypercalcemia and/or hyperphosphatemia refractory to treatment. ${ }^{4,7}$ There has been a trend to consider surgical intervention earlier in the course of SHPT, particularly if the estimated weight of a parathyroid gland exceeds 500 or $1000 \mathrm{mg}$ (normal weight 30 to $40 \mathrm{mg}$ ), and thereby more likely to have nodular hyperplasia. ${ }^{10}$ In addition, large glands that are detectable by ultrasound may be less likely to respond to medical treatment. ${ }^{11}$

While the patient did not present with hypercalcemia, parathyroidectomy was considered the best treatment option because of progressive hyperparathyroid bone disease, persistently elevated PTH and phosphorus levels and unrelenting vitamin D deficiency. In this setting, PTHinduced release of phosphate from bone may have contributed to the persistent hyperphosphatemia, which precludes improvement with vitamin D.

Subtotal parathyroidectomy involves excision of all identifiable parathyroid tissue except for 40 to $60 \mathrm{mg}$ of the least hyperplastic gland. Some surgeons prefer total parathyroidectomy with or without autotransplantation of small amounts of resected parathyroid tissue to reduce the risk of persistent and/or recurrent disease. There have been no randomized trials comparing these techniques. Nevertheless, these procedures are extremely successful in controlling the hyperparathyroidism in the short term. Moreover, long-term follow up demonstrated roughly equal proportions of patients with persistently normal PTH, recurrent hyperparathyroidism and permanent hypoparathyroidism among different techniques. ${ }^{12}$

There is little data regarding the long-term effect of parathyroidectomy on bone healing and risk of fractures. A 3-year longitudinal study demonstrated a marked increase in bone mass after parathyroidectomy among hemodialysis SHPT patients, compared to primary HPT and renal transplant HPT. This demonstrated a 7 to $23 \%$ 
improvement in BMD, which resulted to near restoration to normal from their preoperative bone loss. BMD in the lumbar spine and femoral neck increased by $17 \%$ one year after parathyroidectomy in the hemodialysis SHPT group. This increase was large enough to bring the BMD in the majority of these patients within the reference range of age- and sex-matched controls. ${ }^{13}$

To determine the impact of parathyroidectomy on fracture risk, a population-based study assessed the long-term fracture rates among hemodialysis patients who underwent parathyroidectomy. The study showed a significant $32 \%$ lower risk for hip fracture [95\% CI (0.54 to $0.830), \mathrm{p}=0.001]$ and $31 \%$ lower risk for any analyzed fracture [95\% CI (0.57 to 0.83), p < 0.001] compared with matched control subjects. ${ }^{14}$ Parathyroidectomy also had a positive impact on nutritional status and humoral and cellular immunity. ${ }^{15,16}$

\section{Conclusion}

The initial therapy for SHPT is medical. However, timely surgical therapy should be instituted before refractory SHPT with severe osteitis fibrosa develops in the stage 5 CKD population. Osteitis fibrosa is now an uncommon manifestation of SHPT due to early screening and intervention. Long-standing, unrecognized SHPT leads to debilitating bone disease and fractures which may be more common in the Philippines than previously perceived, due to the high prevalence of CKD.

This case demonstrates a patient with inadequately treated SHPT that became refractory to medical therapy and eventually led to debilitating consequences. Parathyroidectomy was considered beneficial. Careful evaluation and follow up of patients with SHPT is important to identify those who may obtain more benefit from parathyroidectomy to maintain bone health, prevent fractures and likely prolong survival.

\section{References}

1. $\quad 6^{\text {th }}$ National Nutrition and Health Survey (NNHeS): 2003-2004. Food and Nutrition Research Institute, Department of Science and Technology Research and Development Institute. www.fnri.dost.gov.ph (Accessed April 17, 2012).

2. Conchol M. Secondary hyperparathyroidism in chronic kidney disease. US Nephrology 2006;(2):11-3.

3. Hruska KA, Teitelbaum SL. Renal osteodystrophy. N Engl J Med 1995; 333(3):166-74.

4. National Kidney Foundation. K/DOQI clinical practice guideline for bone metabolism and disease in chronic kidney disease. Am J Kidney Dis 2003; 42(4 Suppl 3):S1-S201.

5. Cunningham J, Locatelli F, Rodriquez M. Secondary hyperparathyroidism: pathogenesis, disease progression, and therapeutic options. Clin J Am Soc Nephrol 2011;6(4):913-21.

6. Owda A, Elhwairis H, Narra S et al., Towery H, Osama S. Secondary hyperparathyroidism in chronic hemodialysis patients: prevalence and race. Ren Fail 2003;25(4):595-602.

7. Kidney Disease: Improving Global Outcomes (KDIGO) CKD-MBD Work Group. KDIGO clinical practice guidelines for the diagnosis, evaluation, prevention, and treatment of chronic kidney diseasemineral and bone disorder (CKD-MBD). Kidney Int Suppl 2009;76(113):S1-S130.
8. Gogusev J, Duchambon P, Hory B et al. Depressed expression of calcium receptor in parathyroid gland tissue of patients with hyperparathyroidism. Kidney Int 1997;51(1):328-36.

9. Jameson L, De Groot LJ. Endocrinology: Adult and pediatric. $6^{\text {th }}$ ed. Philadelphia: Saunders Elsevier, 2010;9906-9907.

10. Tominaga Y, Tanaka Y, Sato $\mathrm{K}$ et al. Histopathology, pathophysiology, and indications for surgical treatment of renal hyperparathyroidism. Semin Surg Oncol 1997;13(2):78-86.

11. Katoh N, Nakayama $M$, Shigematsu $T$ et al. Presence of sonographically detectable parathyroid glands can predict resistance to oral pulsed-dose calcitriol treatment of secondary hyperparathyroidism. Am J Kidney Dis 2000; 35(3):465-8.

12. Gagné ER, Ureña $P$, Leite-Silva $S$ et al. Short- and long-term efficacy of total parathyroidectomy with immediate autografting compared with subtotal parathyroidectomy in hemodialysis patients. J Am Soc Nephrol 1992;3(4):1008-17.

13. Abdelhadi M, Nordenström J. Bone mineral recovery after parathyroidectomy in patients with primary and renal hyperparathyroidism. J Clin Endocrinol Metab 1998;83(11):3845-51.

14. Rudser KD, de Boer IH, Dooley A et al. Fracture risk after parathyroidectomy among chronic hemodialysis patients. J Am Soc Nephrol 2007;18(8):2401-7.

15. Yasunaga $\mathrm{C}$, Nakamoto $\mathrm{M}$, Matsuo $\mathrm{K}$ et al. Effects of a parathyroidectomy on the immune system and nutritional condition in chronic dialysis patients with secondary hyperparathyroidism. Am J Surg 1999;178(4):332-6.

16. Tzanno-Martins C, Futata E, Jorgetti V et al. Restoration of impaired T-cell proliferation after parathyroidectomy in hemodialysis patients. Nephron 2000; 84(3):224-7. 